\title{
Alcohol effects on fetal brain development: the case of cynthia
}

\begin{abstract}
The affects of fetal alcohol syndrome disorders continue to affect significant numbers of children in developed nations. Clinical epidemiologist and psychologists have begun to focus on the less obvious but still significant effects often seen in children whose mother drank alcohol during pregnancy. Children exposed to alcohol in utero may not have the facial abnormalities often seen in Fetal alcohol syndrome, but often suffer the same developmental disabilities, including functional central nervous system dysfunction. Alcohol-related neurodevelopmental disorder (ARND) can cause mental, behavioral and learning disabilities, often with lifelong implications. Children suffering from ARND may have a range of disabilities in neurodevelopment and behavior, adaptive skills and selfregulation. What follows is the single case of one such child, Cynthia, born with ARND and continues to struggle today as a teenager with issues of behavior, adaptive skill and self-regulation.
\end{abstract}

Keywords: ARND, fetal, neuropsychology, development, cognitive, memory, behavior, pediatrics, psychotherapy, brain, pregnancy
Volume 2 Issue 2 - 2015

\author{
W Sumner Davis,' Siobhan Crosbie ${ }^{2}$ \\ 'Community Psychologist \& Clinical Epidemiologist, USA \\ ${ }^{2}$ Psychotherapist \& BPS Clinical Supervisor, UK
}

Correspondence: W Sumner Davis, Community Psychologist \& Clinical Epidemiologist, 1 I 2 Allagash Drive, Oakland, Maine 04963, USA, Tel 2077402028,Email drwdavis@mac.com

Received: December 17,2014 | Published: February 16, 2015
Abbreviations: ARND, alcohol-related neurodevelopmental disorder; FAS, fetal alcohol syndrome; KABC, kaufman assessment battery for children; WISC, wechsler intelligence scale for children; IQ, intelligence quotient; MRI, magnetic resonance imaging; PET, positron emission tomography; EEG, electro encephalogram

\section{Introduction}

Cynthia was a precocious 10 -year-old with a quick smile and an infectious laugh. On the surface, she seemed like any other 10-yearold girl. She was interested in all the things that most 10-year-old girls are. She loved to read, although she often chose books that were a little beyond her years and some of her favorites were mysteries and books about vampires. If you were to have met Cynthia, you would probably think that she was like any other typical 10-year-old girl and you would have been right, at least in most respects. Cynthia had great difficulty sitting still, focusing or interacting with her peers. Her adoptive mother, out of concern for Cynthia, had taken her for a number of evaluations. Cynthia had been at different times diagnosed by clinical social workers or psychologists with Anxiety Disorder, Attention Deficit Hyperactivity Disorder, Oppositional Defiant Disorder and Bipolar Disorder. The more professionals she had seen, the more diagnosis she received; all these diagnosis used observable, subjective behavior to render a diagnosis. Yet none of these diagnoses helped Cynthia or helped to explain why she was different. Her problem went back to before school, before infancy even. It began in utero. What separated Cynthia from her classmates was that Cynthia's mother drank alcohol daily while pregnant with Cynthia.

\section{Case presentation}

If you had spent more than a few minutes with Cynthia, you might have noticed that she frequently had difficulty controlling her emotions. She got frustrated easily and that often got her into trouble at school. She had conflicts with the other girls, although she responded well to her teachers. On the Kaufman Assessment Battery for Children (KABC), Cynthia scored 'average' for memory and application tasks, but quite a bit lower than her peers on executive processing tasks. She also had great difficulty with novel tasks and she was easily frustrated. When we read a book together, she often did not understand what she was reading, even though she pronounces the words correctly. If I were to ask her a few minutes later to tell me about what she had read, she remembered what the characters did, but often had difficulty explaining why they did them.

Cynthia's birth mother was a heavy drinker and she did not stop while she was pregnant. As a result, Cynthia's brain was injured before she was born. Children exposed to prenatal alcohol exposure suffer insults to brain structure and function. Studies have shown these brain injuries play a role in a variety of behavioral effects seen later in childhood. Fetal alcohol exposure (ARND) is also associated with deficits in a range of areas of function, including both cognitive functioning which entails general intellectual functioning and the learning of new verbal information, as well as fine and gross motor performance. Neurological studies, particularly those of Berman \& Hannigan ${ }^{1}$ have analyzed the cognitive impairments of children with histories of prenatal alcohol exposure and although many of these studies have focused on children diagnosed with fetal alcohol syndrome (FAS) and analyses have included children with lesser exposures to alcohol in utero. Another study by Clark et al., ${ }^{2}$ show that strong similarities exist between (FAS) children and those exposed to lesser amounts of alcohol in utero or ARND.

\section{Discussion}

As previously mentioned, Cynthia's cognitive ability had been reported as being on the borderline range of normal functioning; her IQ on the standardized tests like the Wechsler Intelligence Scale for Children (WISC), was lower than the range expected for kids her age, but the reason for the lower than expected IQ on such tests are not indicated. Both anecdotal information and the results from studies have demonstrated that this prenatal exposure to alcohol greatly 
affects memory and learning abilities in childhood and observations of children with ARND support this observation. Some studies, ${ }^{2}$ show that children exposed to alcohol in utero demonstrated deficits in memorizing verbal information; this deficit resulted from difficulties with the acquisition of the information rather than the ability to remember the information over time.

Connor \& Mahurin ${ }^{3}$ found that learning deficits occurred in both verbal and nonverbal areas of information acquisition. For parents and teachers, this means that children whose brains were damaged due to teratogens like alcohol have an uphill battle when it comes to learning. These abnormalities are recognized as behaviors that are viewed as negative or disruptive. The behavior further impedes learning and participation in the classroom, the social environment and home-life. Children with ARND appear to be at increased risk for psychiatric disorders, alcohol abuse, drug abuse and other maladaptation. ${ }^{2}$ Children suffering from ARND are also more likely to be hyperactive, disruptive and impulsive. When these behaviors are combined with a diminished capacity for cognitive acquisition, these children are at a great disadvantage in the classroom. Teachers and parents often do not understand that the child is merely using the limited tools they have to try to fit in. Having a learning disability is tough. Having a behavioral disability is tough. Having both makes it nearly impossible to excel in today's 'one-size-fits-all' classrooms. In these surroundings, planning, organizing and forming strategies are all aspects of social grouping that require a group of higher-level cognitive abilities called executive functioning.

Executive functioning is critical to the success of problem-solving, abstract thinking, planning and a flexible thought process. Cognitive abilities can be thought of as tools that the child uses to interpret their world; executive functioning refers to the ability to use these cognitive tools. Children with heavy prenatal alcohol exposure are at risk of impairments on executive functioning tasks. More importantly, a child's deficits in executive functioning are often unrelated to their overall intellectual level, as indicated by studies among adults with ARND. ${ }^{4}$ Moreover, deficits in executive functioning have real-life implications for people exposed to alcohol in utero. They may act without first considering the consequences of their behavior or they may have difficulties linking behavior and consequence. These types of deficits help explain why children with heavy prenatal alcohol exposure, even those with average IQ scores, often have difficulty in school and in society in general.

The neuropsychological and behavioral issues described above manifest themselves in real life effects of prenatal alcohol exposure. The effect of alcohol on brain development has been noted in articles on FAS dating back to the 1970s. With the advent of structural imaging techniques such as MRIs and EEGs as well as imaging scanning devices for example PET and the Single Photon Emission Computed Tomography, researchers can now study the brains of alcohol affected children in non-invasive fashions. ${ }^{2}$ What they're finding strengthens the argument against drinking while pregnant; longitudinal studies now show many complications, both psychological and physiological resulting not only with FAS, but also ARND.

ARND and following on from the example given in the above case study of Cynthia, the child that suffers with behavioral, emotional and educational difficulties resulting from neurological injury in utero are often forced to adopt somewhat antisocial defense mechanisms. This can result in anger becoming a predominant response to others. Children like Cynthia often become vulnerable to the verbal aggression of their peers and respond aggressively. Intellectually they may experience anxiety over the inability to perform educationally compared to peers. This anxiety can manifest as unwanted behaviors and they can be extensive; Cynthia often used anger in communicating with her peers and even some adults. She would attempt to draw attention to herself, for example falling off her chair during class to illicit laughter from her peers. This drew the ire of her teacher and in the long term, such behavior often resulted in consistent rejection that reinforced her feelings of worthlessness. Children like Cynthia may also have greater difficulties in coping with themselves.

Children suffering from ARND are often ill equipped to understand their behavior and may be prone to criticism for behaving in ways that are not deemed socially acceptable or 'normal'. The impact of the child's neurological injury often manifests in struggles with memory or comprehension, which if not handled expertly can result in feelings of low self-esteem, internal anger or overt anger and high levels of anxiety. Feelings of isolation, confusion, frustration and anxiety are too often the result of failed interactions and depending on the level of emotions involved may lead to serious self-harm as a way to deal with the frustration as these children progress to adulthood as a way to control the emotional chaos. This anxiety and need for control may be placed onto food that can result in Bulimia or Anorexia.

These feelings of rejection often turn inward through the child's experiences with rejection from peers or adults from unwanted behavior or being 'slow' educationally or internally by the inability to feel understood or accepted. Anxiety can also manifest in social situations creating a self-perception of being odd or abnormal. The reactions of peers and many adults often result in children like Cynthia feeling rejected, without understanding why, often leading to an extensive self-criticism and further rejection of themselves. Cynthia was referred from one therapist to another creating the perception that she was the problem, one that could not be fixed. As she was referred again and again, Cynthia's feelings of rejection were further reinforced as each person who failed her was seen as a rejection.

In the case of Cynthia, the attachment style to her primary caregiver in infancy was extremely unhealthy. Developmental psychologists considered the first 12 to 18 months of a child's life to be crucial in regards to the child's primary caregiver and that this earliest relationship is foundational for all future relationships. ${ }^{5}$

The first 12 months of Cynthia's life were spent with her birth mother before she was removed from custody and placed in the care of a relative. From the age of one, Cynthia's childhood could be described as average, at least concerning her home life. However, the factors of her negative early attachment combined with ARND led to an avoidance of equal relationships: Cynthia attempted to control her friends and her siblings and once commented to me that a person can only have one friend at a time. On several occasions, I witnessed her becoming very jealous and aggressive toward anyone approaching the person she deemed as her one friend. This controlling behavior will often lead to problems with intimacy and for children born with ARND, these perceptions, both internal and external, often result in avoidance of closeness for fear of rejection. As young adults, these can be manifested in relationships that are unhealthy or destructive, due to a lack of self-esteem or an inability to commit for fear of rejection.

What does seem clear is that children like Cynthia are different. With this recognition comes a type of grieving process where the child must try to come to terms with his or her inability to be like 
everyone else. In children with ARND, the grieving process may be unconscious, reflective of the perceived inability to have emotional stability or security. This often results in further frustration, anger and aggression, which may lead to depression as the child enters adulthood. The angry and frustrated child becomes the depressed teen and we often see an increase in eating disorders, tendencies towards violence or withdrawal and isolation. The neurological, psychological and social effects of ARND can be extensive. Unlike many similar fetal defects, prevention is simple: limit or cease alcohol consumption when pregnant. This can only come through a better awareness of drinking in pregnancy. If you are pregnant do not use alcohol. Check with your pharmacist about prescriptions that may be detrimental to your unborn child. The bottom line is simply this: what you put into your body you are putting it into your unborn child.

\section{Acknowledgements}

None.

\section{Conflict of interest}

The author declares no conflict of interest.

\section{References}

1. Berman RF, Hannigan JH. Effects of prenatal alcohol exposure on the hippocampus: special behavior, electrophysiology, and neuroanatomy. Hippocampus. 2000;10(1):94-110.

2. Clark CM, Li D, Conry J, et al. Structural and functional Brain integrity of fetal alcohol syndrome in nonretarded cases. Pediatrics. 2000;105(5):1096-1099.

3. Connor PD, Mahurin RA. Preliminary study of working memory in fetal alcohol damage using fMRI. Journal of international neuropsychological society. 2001;7(2):206.

4. Archibald SL, Notestine FC, Gamst A, et al. Brain dysmorphology in individuals with severe prenatal alcohol exposure. Dev Med Child Neurol. 2001;43(3):148-154.

5. Ainsworth S, Mary D, Blehar M, et al. Patterns of Attachment: A Psychological Study of the Strange Situation. USA: Lawrence Erlbaum Associates; 1978. 416 p. 